\title{
Alternancia y variación de formas verbales tuteantes y voseantes en el español de santiaguinos: estudio de caso basado en un corpus conversacional
}

\author{
Víctor Fernández-Mallat ${ }^{1}$ \\ Georgetown University
}

\begin{abstract}
Resumen
Está ampliamente aceptado que, en intercambios orales con, por ejemplo, familiares, amigos y colegas de trabajo, los hablantes de la modalidad chilena del español alternan entre formas verbales tuteantes y formas verbales voseantes. En este trabajo, basándome en un corpus de interacciones conversacionales entre familiares y amigos oriundos de Santiago, examino, primero, la frecuencia relativa con la que estas personas recurren al voseo verbal en relación con el tuteo verbal y, segundo, los posibles factores de tipo social y lingüístico que condicionan la variación, prestando particular atención a los parámetros que inciden en el uso del voseo. Igualmente, comparo las distribuciones observadas aquí con las que se observan en estudios previos a este que basan sus corpus en contextos comunicativos menos naturales. Los resultados indican que, en interacciones conversacionales espontáneas, los hablantes recurren al voseo verbal
\end{abstract}

\footnotetext{
1 Para correspondencia, dirigirse a: Víctor Fernández-Mallat (victor.fernandez. mallat@gmail.com), Georgetown University, Department of Spanish and Portuguese, Bunn Intercultural Center 403A, Box 571039, 37th and O Streets, N.W., Washington D.C. 200571039, United States of America.
} 
con una frecuencia mucho mayor que con la que recurren al tuteo verbal y que las frecuencias observadas en estudios previos. Esto, y el hecho de que el voseo esté condicionado por una interacción entre el género y la edad de los hablantes, así como por la especificidad de sus interlocutores, pone en evidencia que los hablantes del español chileno originarios de Santiago asignan al voseo verbal un prestigio encubierto.

Palabras clave: sociolingüística, variación, alternancia tuteto $\sim$ voseo, español, Santiago de Chile.

\title{
Alternation AND VARIATION OF VERBAL TUTEO AND VERBAL VOSEO IN THE SPANISH OF SANTIAGUINOS: A CASE STUDY BASED ON A CONVERSATIONAL CORPUS
}

\begin{abstract}
It is widely accepted that in oral exchanges with, for example, family members, friends, and co-workers, speakers of Chilean Spanish alternate between verbal tuteo and verbal voseo. In this paper, drawing on a corpus of conversational interactions between relatives and friends from Santiago, I examine the relative frequency with which these speakers use verbal voseo in relation to verbal tuteo, and I explore the social and linguistic factors that determine the variation, paying particular attention to the parameters that affect the use of verbal voseo. In addition, I compare the distributions observed here with those observed in previous studies that base their analyses on corpora that contain less natural communicative contexts. The results indicate that, in spontaneous conversational interactions, the speakers use verbal voseo with a frequency much greater than the frequency with which they use verbal tuteo as well as the frequencies observed in previous studies. This, and the fact that verbal voseo is conditioned by an interaction between the gender and the age of the speakers, as well as by the specificity of their interlocutors, shows that speakers of Chilean Spanish born in Santiago assign covert prestige to verbal voseo.
\end{abstract}

Keywords: Sociolinguistics, variation, tuteo $\sim$ voseo alternation, Spanish, Santiago de Chile.

Recibido: 14/07/17 Aceptado: 30/04/18 


\section{INTRODUCCIÓN ${ }^{2}$}

El grueso de los estudios sobre las formas de tratamiento en uso en el español chileno actual (p. ej., Helincks 2015; Rivadeneira y Clua 2011; Torrejón 2010b) coinciden en que, en situaciones de inmediatez comunicativa ( $p$. ej., en intercambios orales entre familiares, amigos y colegas de trabajo), los hablantes de esta modalidad del español alternan entre formas verbales tuteantes y formas verbales voseantes, mientras que, en situaciones de distancia comunicativa (p. ej., en relaciones jerárquicas como la que puede darse entre un empleado y su empleador), los hablantes recurren preferentemente a formas verbales ustedeantes ${ }^{3}$.

En lo que concierne específicamente al sistema voseante del español chileno, la mayoría de los estudios recientes (p. ej., Fernández Mallat 2011; Rivadeneira Valenzuela 2016; Torrejón 2010a) está de acuerdo con el hecho de que se trata esencialmente de un sistema mixto verbal, término acuñado por Torrejón (1986) para referir a aquellos paradigmas verbales en los que los hablantes combinan desinencias voseantes de la segunda persona del singular con el pronombre personal $t u^{4}$.

Respecto a la morfología de las formas verbales voseantes, los últimos estudios (p. ej., Bishop y Michnowicz 2010; Rivadeneira Valenzuela 2016; Stevenson 2007) coinciden en que, en Chile, estas formas gramaticales corresponden al voseo de tipo III de Rona (1967), que, en el presente de indicativo, consiste en mantener formas verbales diptongadas para los verbos de la primera conjugación (p. ej., hablái) y en adoptar formas verbales en

\footnotetext{
2 Quisiera expresar mi agradecimiento a las personas que compartieron parte de su tiempo conmigo y que, de esta manera, permitieron que este trabajo se realizara.

3 Es de notar que los hablantes de la modalidad chilena del español también recurren al ustedeo en ciertas situaciones de inmediatez comunicativa, como por ejemplo cuando una madre le habla a su hijo con ternura (Rivadeneira Valenzuela 2016: 93). No obstante, el paradigma más corriente para la segunda persona del singular es el que incluye formas verbales tuteantes y voseantes para situaciones de inmediatez comunicativa y formas verbales ustedeantes para situaciones en las que prima la distancia comunicativa.

4 Esto no significa que no se encuentren en el habla de personas de origen chileno instancias de voseo auténtico, término también acuñado por Torrejón (1986) para referir a aquellos paradigmas verbales en los que los hablantes combinan desinencias voseantes de la segunda persona del singular con el pronombre personal vos. No obstante, su uso está más bien limitado a situaciones comunicativas de descortesía (Torrejón 2010b: 765) o a situaciones comunicativas en las que hablantes jóvenes de sexo masculino quieren marcar pertenencia grupal o solidaridad con miembros del grupo con el que se identifican (Rivadeneira Valenzuela 2016: 93).
} 
las que se monoptonga la vocal más cerrada para los verbos de la segunda y tercera conjugaciones (p. ej., sabíh y vivíh). En términos de realización fonética, existe una marcada tendencia a elidir la /-s/ de las formas verbales diptongadas, mientras que con las formas verbales monoptongadas la tendencia es más bien a la aspiración (Torrejón 2010a: 415).

Varios estudios (p. ej., Newall 2007; Rivadeneira Valenzuela 2016; Sweeney 2005) concuerdan en que la estructura mixta del voseo verbal chileno y la alternancia tuteo voseo que se observan en la actualidad en Chile pueden explicarse con base en un hecho histórico: la reforma gramatical que propició el polímata Andrés Bello poco después de haberse instalado en Santiago de Chile con su familia, a mediados del siglo XIX. En esta reforma, entre otras cosas, el intelectual de origen venezolano caracterizaba el voseo como un "peca[do] contra el buen uso" (Bello 1884 [1834]: 469). Como lo señala Torrejón (1989: 547), antes de ese evento histórico, el voseo auténtico era en Chile el modo universal de tratamiento en situaciones en las que primaba la inmediatez comunicativa. Después de dicho evento, tal y como lo indica Newall (2007: 168-169), el voseo adoptó un marcado estigma social y, en consecuencia, su uso disminuyó considerablemente a favor del tuteo. En lo que toca a la emergencia de su estructura mixta verbal, Bertolotti (2015: 19) sugiere que, al ser las categorías léxicas como el pronombre personal vos más perceptibles por los hablantes que las categorías gramaticales como las formas verbales voseantes, su mantenimiento en las desinencias se vio ampliamente facilitado.

La estigmatización del voseo en Chile es perceptible hasta el día de hoy. Una muestra de ello radica en el hecho de que, en función del método mediante el cual se lleve a cabo la recogida de datos, los estudios actuales obtienen distintos resultados en lo que concierne a la frecuencia relativa con la que los hablantes utilizan o piensan utilizar soluciones verbales voseantes en comparación con soluciones tuteantes. En datos obtenidos a partir de cuestionarios de actitudes lingüísticas (Bishop y Michnowicz 2010) e interacciones conversacionales desarrolladas en programas radiales (Rivadeneira y Clua 2011) y televisivos (Torrejón 2010b), se puede apreciar una tendencia a preferir las formas tuteantes sobre las formas voseantes. En cambio, de acuerdo con datos obtenidos con base en notas de campo (Bishop y Michnowicz 2010) y entrevistas sociolingüísticas (Rivadeneira Valenzuela 2016), se puede observar la tendencia contraria. En el Gráfico 1, a continuación, se ilustran las distintas distribuciones de la variable tuteo voseo en función del método mediante el cual se lleva a cabo la recogida de los datos. 
Gráfico 1: Distribución de la variable tuteo voseo según el método de recogida de datos

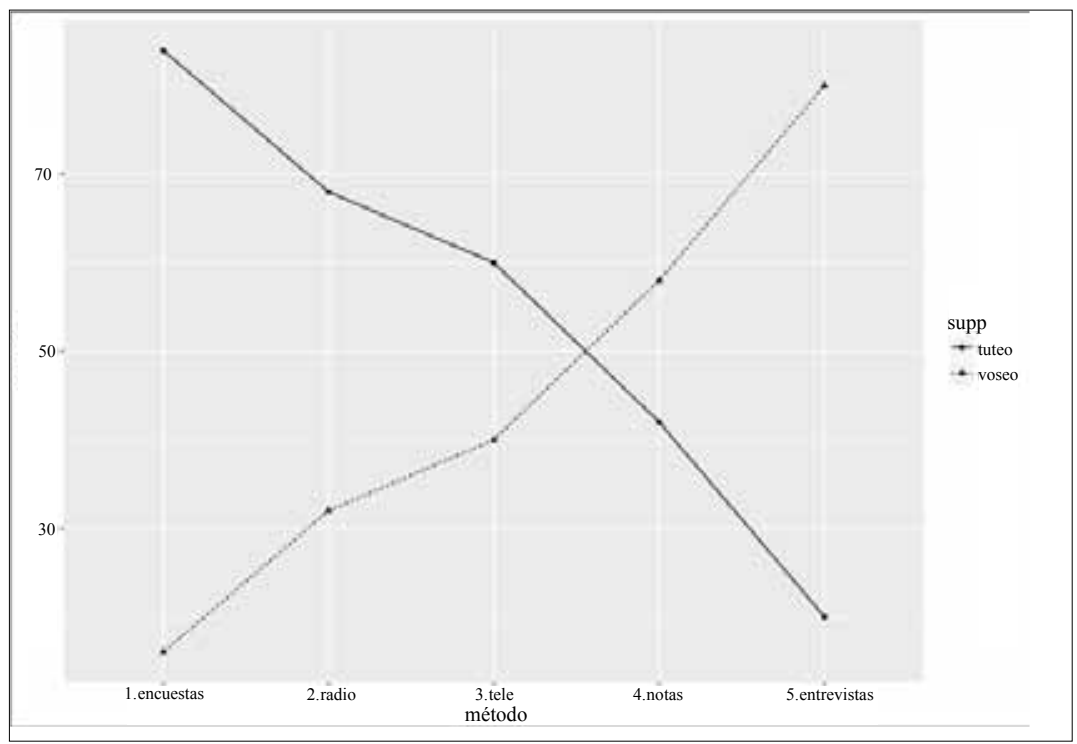

Esta distribución, que pone de manifiesto que, en los contextos lingüísticos más naturales, los hablantes prefieren notoriamente las formas verbales voseantes sobre las formas verbales tuteantes, mientras que en los contextos lingüísticos menos naturales o en reflexiones metalingüísticas se observa la preferencia contraria, corresponde a la pauta clásica de prestigio encubierto que describen, por ejemplo, Labov (1966) y Trudgill (1972) para el mundo anglófono y Silva-Corvalán (1984) para el mundo hispanohablante.

Otra muestra de la estigmatización del voseo verbal en Chile radica en su distribución desigual en función de parámetros sociales y lingüísticos. Entre los factores sociales que se han asociado con la alternancia tuteo voseo se ha identificado el género y la edad. Bishop y Michnowicz (2010) encuentran una correlación positiva entre una propensión a usar las formas voseantes y las hablas masculina y juvenil, lo cual también corresponde a la pauta clásica de prestigio encubierto que autores como Labov (1966) Trudgill (1972) y Silva-Corvalán (1984) describen. Rivadeneira y Clua (2011) y Rivadeneira Valenzuela (2016) han confirmado este hallazgo en estudios posteriores ${ }^{5}$.

5 De manera interesante, Carvalho (2010), quien estudia la alternancia tuteo $\sim$ voseo en el español fronterizo uruguayo, también encuentra conexiones entre dicha alternancia y los 
En lo que atañe a los parámetros lingüísticos que inciden en la alternancia tuteo voseo de manera considerable, se ha identificado la especificidad del interlocutor. Kluge (2005) observa que hay una tendencia a usar las formas verbales voseantes con cierta reserva en el tratamiento directo de las personas involucradas en la conversación y que esta reserva es inexistente cuando las formas verbales voseantes se usan para hacer referencia a experiencias de vida generalizables a la experiencia de más personas. Fernández Mallat (2011) ha confirmado este hallazgo de manera más reciente. Sostengo aquí que el hecho de que las formas verbales voseantes estén estigmatizadas entre los hablantes del español chileno puede explicar en cierta medida los hechos observados por estos autores 6 .

En el presente estudio, me propongo dos objetivos centrales. Primero, me planteo comparar la frecuencia relativa con la que los hablantes del español chileno oriundos de Santiago recurren al voseo verbal en relación con el tuteo verbal en el corpus conversacional que sirve de base a esta investigación con las frecuencias relativas que se observan a partir de los cuestionarios de actitudes lingüísticas de Bishop y Michnowicz (2010), las interacciones conversacionales desarrolladas en programas radiales y televisivos de Rivadeneira y Clua (2011) y Torrejón (2010b), respectivamente, las notas de campo de Bishop y Michnowicz (2010) y las entrevistas sociolingüísticas de Rivadeneira Valenzuela (2016). Debido a la naturaleza conversacional del corpus que empleo en este estudio, anticipo obtener frecuencias relativas de uso del voseo verbal más altas que las que se han obtenido en todos los trabajos citados arriba. En efecto, sobre la base de lo que se observa en estudios como los de Labov (1966), Trudgill (1972) y Silva-Corvalán (1984),

factores del género y de la edad. Para la edad, al igual que Bishop y Michnowicz (2010), Rivadeneira y Clua (2011) y Rivadeneira Valenzuela (2016), Carvalho (2010: 14) nota una correlación positiva entre una tendencia al voseo y el habla juvenil, lo cual interpreta como un posible cambio en curso a favor de las formas voseantes. Para el género, a diferencia de los autores recién citados, Carvalho (2010: 15) observa una correlación positiva entre una tendencia al voseo y el habla femenina. La autora (2010: 15) interpreta el hecho de que las personas que se identifican con el género femenino prefieran las formas voseantes de manera significativamente más alta que las que se identifican con el género masculino como una señal de que este tipo de formas goza de prestigio entre los hablantes de la variedad fronteriza del español uruguayo. En este sentido, el estatus del voseo en esta modalidad se distingue del estatus que tiene en lo hasta aquí observado para el español chileno.

6 Es notable que Carvalho (2010: 12) observe para el español fronterizo uruguayo lo mismo que Kluge (2005) y Fernández Mallat (2011) para el español hablado por personas de origen chileno. Este hecho deja suponer que, a pesar del cambio en curso que la autora nota en función de los parámetros sociales que inciden en el uso del voseo, este rasgo sigue estando socialmente marcado en el español hablado en el norte uruguayo. 
lo esperable sería encontrar una frecuencia relativa de uso del voseo verbal en relación con el tuteo verbal aún más alta que la frecuencia del $80 \%$ que indica Rivadeneira Valenzuela para la ciudad de Valparaíso (2016: 102) ${ }^{7}$, ya que el contexto conversacional en el que se desarrollan los intercambios que analizo en el corpus que sirve de base a este estudio es aún más natural que el contexto que se da en las entrevistas sociolingüísticas conducidas por esta investigadora.

En segundo lugar, me propongo identificar los posibles parámetros sociales y lingüísticos que condicionan considerablemente la alternancia tuteo $\sim$ voseo en contexto conversacional. Sobre la base de las observaciones de Bishop y Michnowicz (2010), Fernández Mallat (2011), Kluge (2005), Rivadeneira y Clua (2011) y Rivadeneira Valenzuela (2016), anticipo hallar correlaciones significativas entre dicha alternancia y los factores del género, la edad y la especificidad del interlocutor. De manera específica, preveo que las personas de género femenino y en la primera etapa de su vida adulta laboral usen las formas verbales voseantes con cierta reserva en comparación con las personas de género masculino y en otras etapas de su vida. En cuanto a la especificidad del interlocutor, anticipo que todos los hablantes muestren cierta reserva a usar el voseo verbal para el tratamiento directo de sus interlocutores en comparación con el uso que hacen de esta forma para referir a aquellas experiencias de vida que se pueden generalizar a la experiencia de otras personas. No descarto el hecho de que otros parámetros sociales o lingüísticos puedan llegar a influir significativamente en la alternancia tuteo voseo; por eso, como se verá en la próxima sección, los incluyo en mi análisis.

\footnotetext{
Es de notar que este porcentaje de uso fue calculado excluyendo la consideración que Rivadeneira Valenzuela (2016) hace del ustedeo en su estudio, ya que el foco de atención del presente trabajo es exclusivamente el de la alternancia tuteo $\sim$ voseo. Igualmente, cabe destacar que este porcentaje corresponde a lo observado por Rivadeneira Valenzuela (2016: 102) para la ciudad de Valparaíso, a pesar de que esta autora incluya en su estudio datos procedentes de otras localidades, concretamente de Iquique, Temuco y Coyhaique, que se ubican en los extremos norte y sur del país. Opto por excluir los datos procedentes de estas tres ciudades porque se ha comprobado que el uso del voseo en la zona central de Chile, que incluye a ciudades como Valparaíso y Santiago (de donde provienen mis datos), es mayor que en otras zonas del país (Rivadeneira y Clua 2011: 691; Rivadeneira Valenzuela 2016: 106). En este sentido, los datos procedentes de Iquique, Temuco y Coyhaique no se prestan a comparación con los que uso aquí.
} 


\section{MÉTODOS}

El corpus que sirve de base a este estudio está constituido por una serie de conversaciones informales y espontáneas grabadas entre los años 2012 y 2014 con 20 familiares, amigas y amigos. De todos los hablantes, 9 se identifican con el género femenino y 11 con el género masculino. Sus edades van desde los 24 hasta los 54 años. Todos han nacido y crecido en Santiago de Chile, donde viven, y tienen el español como lengua materna. En las grabaciones, que tienen una duración que va desde los 60 hasta los 90 minutos, siempre hay tres o más hablantes interactuando.

Para el análisis de los datos, adopto un enfoque variacionista (Bayley 2013; Tagliamonte 2012). Por este motivo, solo tomo en consideración aquellas formas verbales que contienen variantes tuteantes y voseantes. Así pues, de todas las formas verbales de tratamiento para referir a la segunda persona del singular presentes en las grabaciones, excluyo toda ocurrencia del marcador discursivo cachái porque se trata de una forma que está plenamente lexicalizada en el habla chilena (Fernández Mallat 2011: 53; Rivadeneira Valenzuela 2016: 100). También descarto toda ocurrencia de verbos conjugados en tiempos verbales que no presentan flexión voseante, como el pretérito perfecto simple de indicativo, el futuro simple de indicativo y de subjuntivo y el imperativo (Fernández Mallat 2011: 55; Rivadeneira Valenzuela 2016: 91).

Siguiendo la tradición variacionista, codifico las ocurrencias que extraigo de las grabaciones en función de factores sociales y lingüísticos para identificar aquellas variables independientes que condicionen la distribución de formas tuteantes y voseantes en el habla de las personas grabadas de manera significativa. Analizo las ocurrencias usando modelos lineales generalizados mixtos con los paquetes informáticos lme4 (Bates et al. 2015) y nlme (Pinheiro et al. 2017) en el lenguaje de programación $\mathrm{R}$ (R Development Core Team 2017).

Los parámetros sociales en función de los cuales codifico los datos son el género y la edad de los hablantes. Tengo en cuenta estos dos factores extralingüísticos por la importancia que tienen en determinar la distribución de formas verbales tuteantes y voseantes en estudios recientes previos a este (p. ej., Bishop y Michnowicz 2010; Rivadeneira y Clua 2011; Rivadeneira Valenzuela 2016). No considero la clase social como criterio de codificación por dos motivos. Primero, se trata de un factor extralingüístico cuya insignificancia en condicionar la distribución de formas verbales tuteantes y voseantes se demuestra en varios estudios actuales (p. ej., Bishop y 
Michnowicz 2010; Helincks 2012; Rivadeneira y Clua 2011) y no tan actuales (p. ej., Morales 1972-1973; Torrejón 1986). Segundo, al proceder los datos con los que trabajo de conversaciones grabadas con familiares y amistades, la ubicación de clase de todas las personas cuyas voces fueron grabadas es grosso modo de clase media.

Para el género, como se dijo, cuento con datos procedentes del habla de 9 personas que se identifican con el género femenino y 11 personas que se identifican con el género masculino. Para la edad, basándome en autores como Eckert (1997) y Wolfram, Hazen y Schilling-Estes (1999), adopto un enfoque émico en función del cual agrupo a los hablantes en personas que están recién empezando su vida laboral y que no tienen puestos de trabajo seguros y en personas que ya tienen bastante experiencia en la vida laboral y que tienen puestos de trabajo relativamente seguros. En el primer grupo, que llamo adulto joven para los propósitos de este estudio, cuento con datos procedentes del habla de 11 personas que tienen entre 24 y 32 años. En el segundo grupo, que llamo arbitrariamente adulto, cuento con datos procedentes del habla de 9 personas cuyas edades van desde los 38 hasta los 54 años.

Los factores lingüísticos a partir de los cuales codifico los datos son el pronombre personal que precede al verbo, la especificidad del interlocutor, el paralelismo lingüístico, el tiempo verbal y la forma verbal usada por el interlocutor. Tomo en consideración estos factores internos basándome en el hecho de que inciden significativamente sobre la distribución de formas voseantes en estudios previos a este, no solo sobre el español chileno ( $p$. ej., Kluge 2005; Fernández Mallat 2011; Rivadeneira y Clua 2011), sino que también sobre el español fronterizo uruguayo (p. ej., Carvalho 2010).

Para el pronombre personal que precede al verbo, si bien las posibilidades observadas son el uso de vos, el uso de tú y el uso del sujeto tácito (p. ej., Torrejón 2010a: 416; Rivadeneira Valenzuela 2016: 90), en este estudio me limito a tener en cuenta las dos últimas posibilidades (1a y $1 \mathrm{~b}$, respectivamente), ya que son las únicas que se dan en mi corpus.

\section{(1) a. tú conocíh al tío tito poh \\ b. Ø teníh que agarrarlo al tiro}

En lo que atañe a la especificidad del interlocutor, considero la posibilidad de que el hablante use la segunda persona del singular en el tratamiento directo de sus interlocutores (2a), en el tratamiento directo reproducido (2b) o para hacer referencia a experiencias de vida generalizables a la experiencia de más personas $(2 \mathrm{c})$. 
(2) a. lo que tú mencionái (.) que tiene que ver (.) precisamente con eso

b. me los pasa y me dice defiéndelos (.) ve tú cómo los defendíh

c. después (.) al final del carnaval (.) quedái toda ronca

Con respecto al paralelismo lingüístico, que consiste en la tendencia que tiene una forma lingüística a ocurrir repetidamente en el marco de una secuencia discursiva (Carvalho 2010: 9), estudio la posibilidad de que la forma verbal que use un hablante sea la primera forma de la secuencia (3a o el primer verbo conjugado en la segunda persona del singular de los ejemplos 3 b y $3 c$ ) o la que, dentro de la misma secuencia, siga a una forma voseante (3b). Si bien, tal y como lo indica Carvalho (2010: 9), también existe la posibilidad de que, dentro de una secuencia, una forma verbal de tratamiento siga a una forma tuteante, excluyo esta alternativa, ya que, en el corpus que analizo en este estudio, las nueve ocurrencias que corresponden a dicho contexto son siempre formas verbales tuteantes (3c). En otras palabras, son ocurrencias que no presentan alternancia tuteo $\sim$ voseo.

(3) a. al final (.) tú (.) por ejemplo (.) podíh tener bajas notas

b. y pegái en el cárter (.) reventái el cárter del motor

c. si tú sales (.) por ejemplo (.) tienes que esperar un día

En lo que se refiere al tiempo verbal, tomo en consideración solo aquellos paradigmas flexivos que se dan en el corpus que sirve de base a esta investigación. Para el modo indicativo, tengo en cuenta el presente (4a), el pretérito perfecto compuesto (4b), el pretérito imperfecto (4c), el pretérito pluscuamperfecto (4d) y el condicional simple (4e). Para el modo subjuntivo, tomo en consideración el presente (4f), el pretérito imperfecto $(4 \mathrm{~g})$ y el pretérito pluscuamperfecto (4h) de subjuntivo.

(4) a. prométeme que si te falta plata huevón me decíh ya (.) necesito plata

b. no sé si tú hai tenido la oportunidad de ir a alguna fiesta

c. tú hacíai queso de cabeza cuando (.) bueno (.) alguna vez

d. cómo y por qué habíai aprendido a hablar inglés

e. eso deberíai escribir (.) una tesis sobre la ironía huevón

f. cuidate (.) chao (.) que estíh muy bien

g. hablante 1: entonces te castigaban (.)

hablante 2: aunque vivierai en zona extrema

h. yo quería que (.) puta (.) hubierai incluido esa huevada 
En lo que incumbe a la forma verbal usada por el interlocutor, considero la posibilidad de que este no use una forma de tratamiento para referirse a la segunda persona del singular (5a), que recurra al tuteo verbal (5b) o, por último, que use el voseo verbal $(5 \mathrm{c})$.

(5) a. hablante 1: cómo era el bus (.) de estas huevadas (.) hablante 2: no (.) sabíh que no

b. hablante 1: no puedes decir que no hablante 2: no podíh decirlo

c. hablante 1: estái cagado hablante 2: \{risas\} estái cagado

\section{RESULTADOS}

Los datos procedentes del corpus que sirve de base a este estudio indican que los hablantes recurren a formas verbales voseantes en $87 \%$ de los casos, mientras que usan formas verbales tuteantes en $13 \%$ de los casos. La Tabla 1, a continuación, ilustra la distribución aquí observada, incluyendo ahí el número total de ocurrencias observadas $(\mathrm{N})$.

Tabla 1: Distribución de la variable tuteo voseo en el corpus de estudio

\begin{tabular}{lll} 
Forma & Frecuencia absoluta $(\mathrm{N})$ & Frecuencia relativa \\
\hline Tuteo verbal & $50 / 386$ & $13 \%$ \\
Voseo verbal & $336 / 386$ & $87 \%$ \\
\hline
\end{tabular}

En lo que concierne a los parámetros sociales y lingüísticos que condicionan la alternancia tuteo $\sim$ voseo, el análisis estadístico revela que, en el corpus conversacional que estudio en el marco del presente trabajo, las variables independientes que inciden en el uso de formas verbales voseantes de manera estadísticamente significativa son la especificidad del interlocutor y una interacción de los factores del género y de la edad. La Tabla 2, a continuación, da cuenta de la situación observada. 
Tabla 2: Factores que inciden sobre el uso del voseo (el factor del paralelismo lingüístico se excluye del análisis)

\begin{tabular}{|c|c|c|c|c|}
\hline & Coeficiente & Error estándar & Valor de $z$ & Valor de $p$ \\
\hline (Intercepto) & 2.45 & 0.53 & 4.67 & $<0.01$ \\
\hline \multicolumn{5}{|c|}{$\begin{array}{l}\text { Especificidad del interlocutor (la categoría de referencia es experiencias de vida } \\
\text { generalizables a la experiencia de más personas) }\end{array}$} \\
\hline Tratamiento directo & -1.06 & 0.43 & -2.44 & 0.01 \\
\hline $\begin{array}{l}\text { Tratamiento directo } \\
\text { reportado }\end{array}$ & -1.41 & 0.69 & -2.06 & 0.04 \\
\hline \multicolumn{5}{|l|}{ Interacción (género y edad) } \\
\hline Femenino y adulto joven & -2.76 & 1.29 & -2.14 & 0.03 \\
\hline
\end{tabular}

Como se puede ver, la probabilidad de que los hablantes recurran a formas verbales voseantes en el tratamiento directo de sus interlocutores o en el tratamiento directo reportado es significativamente menor que la probabilidad de que usen estas formas de tratamiento para referir a experiencias de vida que se pueden generalizar a la experiencia de más personas. Igualmente, la probabilidad de que las personas de género femenino que están recién empezando su vida laboral y que no tienen puestos de trabajo seguros usen formas verbales voseantes es significativamente menor que la probabilidad de que las usen las personas de género masculino en la misma etapa de sus vidas o las personas de género femenino o masculino que ya tienen bastante experiencia en la vida laboral y que tienen puestos de trabajo relativamente seguros. En el Gráfico 2, a continuación, se ilustra dicha interacción. 
Gráfico 2: Efecto de la interacción género y edad sobre el uso de formas verbales voseantes

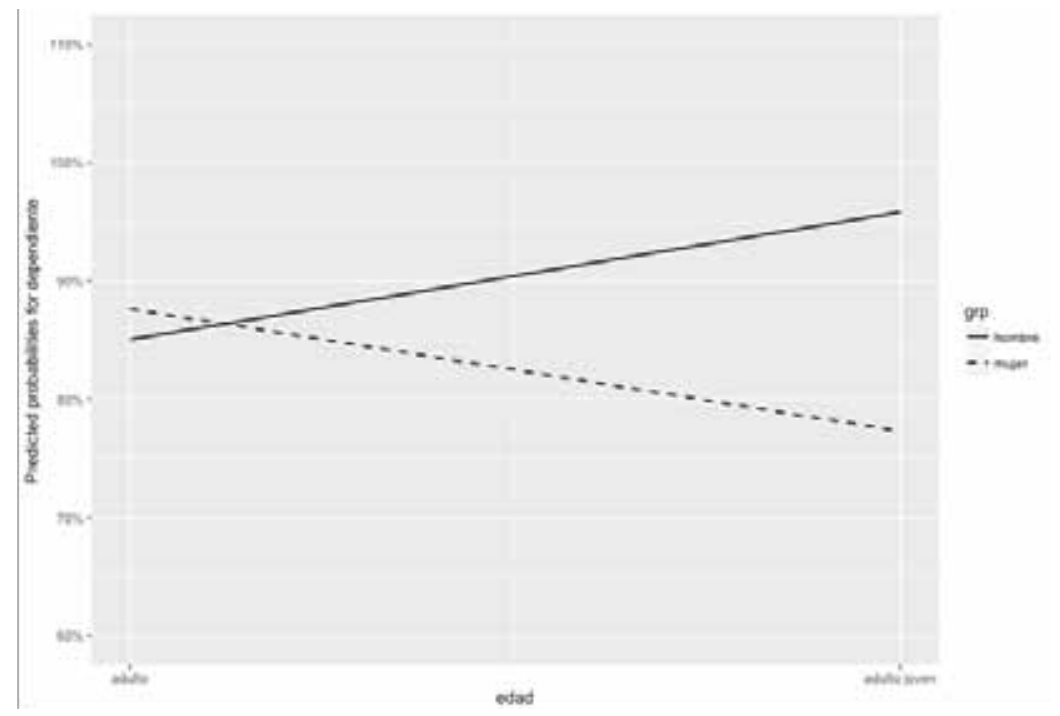

\section{DISCUSIÓN}

En lo que atañe a la frecuencia relativa con la que las personas cuya habla se analiza en este estudio hacen uso de formas verbales voseantes en relación con formas verbales tuteantes, los resultados no son sorprendentes. Tal y como se esperaba, en el contexto más natural de la conversación, la frecuencia relativa con la que los hablantes recurren al voseo verbal es más alta que las frecuencias relativas observadas en estudios cuyos corpus se basan en entrevistas sociolingüísticas (Rivadeneira Valenzuela 2016), notas de campo (Bishop y Michnowicz 2010) e interacciones conversacionales desarrolladas en programas televisivos (Torrejón 2010b) y radiales (Rivadeneira y Clua 2011), que constituyen todos contextos conversacionales menos naturales. En comparación con la percepción de los propios hablantes en lo que se refiere a la frecuencia relativa con la que recurren al voseo verbal (Bishop y Michnowicz 2010), las diferencias observadas en el marco de este estudio tampoco son sorprendentes. Al contrario, ponen de manifiesto que aquello 
que los hablantes creen o piensan hacer es enteramente lo opuesto de lo que hacen en realidad. En el Gráfico 3, a continuación, se ilustran las distintas distribuciones de la variable tuteo voseo en función del método con el que se lleva a cabo la recogida de datos, incluyendo ahí la distribución procedente de los datos analizados en el presente trabajo.

Gráfico 3: Distribución de la variable tuteo voseo según el método de recogida de datos (incluye los datos conversacionales que sirven de base a este estudio)

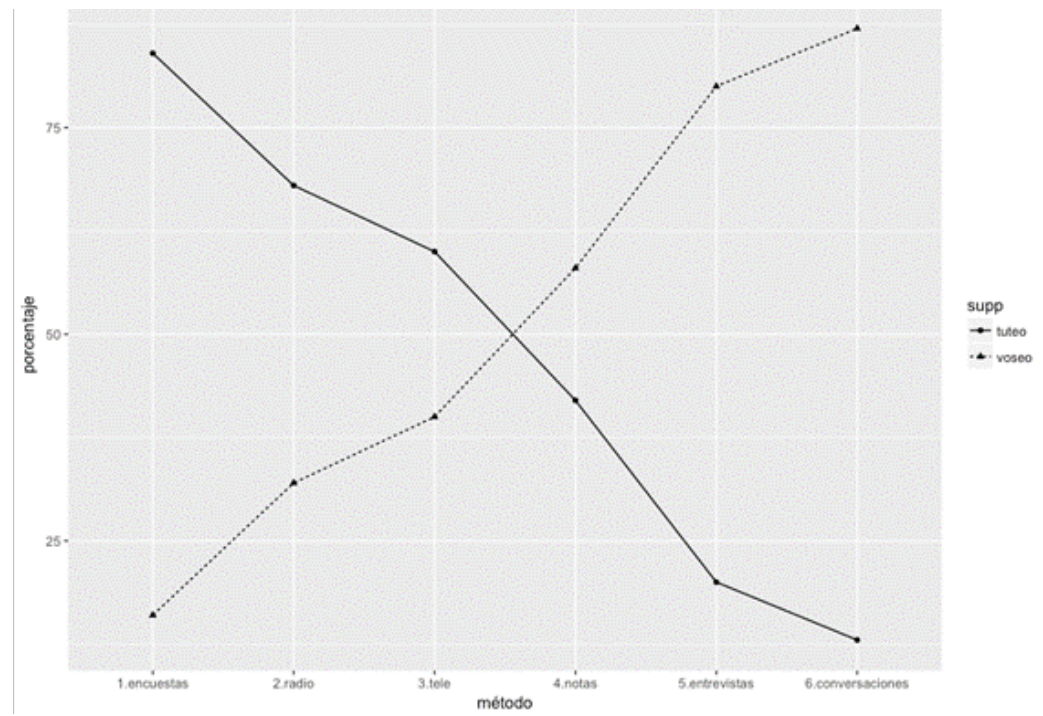

Lo que se observa en el Gráfico 3 al comparar las frecuencias referidas en este y en otros estudios es de interés, puesto que permite confirmar con datos nuevos y conversacionales la idea de autores como Bishop y Michnowicz (2010) y Helincks (2012) según la cual el voseo verbal, aunque sea mixto y, como lo sugiere Bertolotti (2015: 19), menos perceptible que las soluciones voseantes de tipo pronominal, es un rasgo al que los hablantes de la modalidad chilena de español atribuyen un prestigio encubierto.

A continuación, argumento que los resultados observados en lo que respecta a los parámetros sociales y lingüísticos que condicionan el uso del voseo verbal entre personas de origen chileno, tanto aquí como en estudios previos, refuerzan esta idea.

En lo concerniente a la interacción de los factores sociales del género y de la edad, que según el análisis que se hizo es el único parámetro social con un peso estadístico significativo, es preciso señalar que se ha observado repetidamente en la literatura especializada que las personas de género 
femenino tienden a evitar aquellos rasgos lingüísticos que están socialmente estigmatizados (p. ej., Díaz-Campos 2014: 38; Labov 1972: 243). Asimismo, se ha notado frecuentemente que los adolescentes y los adultos mayores suelen usar este tipo de rasgos con mayor libertad o menor contención que aquellas personas que se encuentran en las primeras etapas de su adultez ( $p$. ej., Milroy y Gordon 2003: 39; Coulmas 2005: 61). El hecho de que en el presente estudio se observe que la probabilidad de que las personas de género femenino en una etapa temprana de su vida adulta recurran al voseo verbal es notablemente más baja que la probabilidad de que lo usen sus coetáneos de género masculino y las personas mayores, sin importar su género, está de acuerdo con lo recién señalado.

Dicho esto, es necesario que estudios futuros se centren en el habla de jóvenes adolescentes de género femenino, esto es, de jóvenes chilenas o santiaguinas que se encuentren en una etapa de sus vidas en la que aún no han incorporado el mercado laboral. En el presente estudio, no cuento con datos procedentes de personas con esas características. Un análisis detallado de la frecuencia relativa con la que recurren al voseo verbal y de los factores sociales que condicionan su uso sería esclarecedor en relación con lo aquí observado. En efecto, de confirmarse la idea según la cual la probabilidad de que las adolescentes chilenas o santiaguinas usen el voseo verbal es mayor que la probabilidad de que lo usen sus congéneres en los albores de su vida laboral y similar a la probabilidad de que recurran a él sus congéneres ya avanzadas en sus actividades laborales, podría afirmarse con mayor certeza que los hablantes del español chileno le atribuyen al voseo un prestigio encubierto, puesto que se obtendría evidencia según la cual, en comparación con personas que se identifican con otro género y/o que están en otras etapas de su vida, solo las personas de género femenino al principio de sus actividades laborales muestran una reserva estadísticamente significativa al usar el voseo.

Asimismo, el hecho de observar una estratificación por edad y mayor uso del voseo entre las hablantes chilenas o santiaguinas en la adolescencia y las hablantes de ese origen en una etapa de sus actividades laborales ya avanzada, versus un menor uso de esta forma verbal entre las chilenas en los albores de su vida profesional, permitiría sugerir que el cambio en curso observado por investigadoras como Helincks (2015: 92) y Rivadeneira Valenzuela (2016: 110) en lo que toca a la variación en los usos voseantes de las personas de origen chileno se halla relativamente estabilizado. En efecto, dada esta situación, las curvas observadas en lo que concierne a la frecuencia con la que las hablantes chilenas usan el voseo presentarían una forma similar a una $u$ o a una $v$. Autores como Holmes (1992) y Labov (2001) consideran este tipo de curvas como una señal de que no hay un cambio en 
curso y de que la variación en el uso del rasgo observado es más o menos estable (age-graded variation).

En lo que respecta a la especificidad del interlocutor, que según el análisis que se llevó a cabo aquí es la única variable lingüística con significancia estadística, es preciso señalar que, al usar las formas verbales de la segunda persona del singular en el tratamiento directo, uno se refiere precisamente al interlocutor de la interacción conversacional. En otras palabras, se lo está interpelando de manera directa. Lo mismo se produce al usar estas formas verbales en el tratamiento directo reproducido, con la diferencia de que, en tal caso, uno representa el momento en el que una persona se refiere de modo preciso a su interlocutor. En cambio, al usar una forma de tratamiento para referirse a experiencias de vida que pueden generalizarse a la experiencia de vida de otras personas, uno no tiene como referente directo a su interlocutor, sino que, tal y como lo señala Kluge (2010: 1111), a cualquier persona que se encuentre en la situación a la que se alude en el acto de habla, incluso a uno mismo. En otras palabras, no se está interpelando directamente al interlocutor de la interacción conversacional. El hecho de que, en el presente estudio, así como en otros previos a este, se note que la probabilidad de que los hablantes recurran a formas verbales voseantes en ambos tipos de tratamiento directo sea considerablemente más baja que la probabilidad de que recurran a ellas cuando se refieren a experiencias de vida generalizables a la experiencia de más personas pone de manifiesto que, en el marco del primer contexto comunicativo, los hablantes usan el voseo con mayor reserva o cautela que en el segundo de estos contextos. Con base en que, en el primer contexto, se interpela directamente a su interlocutor, mientras que, en el segundo, no se lo interpela de esta manera, sostengo aquí que este hecho está en línea con la caracterización del voseo verbal como un rasgo que se enfrenta a cierta estigmatización por parte de los hablantes del español chileno.

Por último, es necesario dedicar unas líneas a la variable independiente del paralelismo lingüístico. Como ya se señaló, es un parámetro que excluí del análisis estadístico, ya que una de sus variantes, la posibilidad de que, dentro de una secuencia, la forma verbal que sigue a una forma tuteante sea o bien una ocurrencia de tuteo o bien una de voseo, no presenta variación, sino que, todo lo contrario, muestra contener solo formas verbales tuteantes. Las dos otras posibilidades, esto es, el hecho de que la forma verbal que use un hablante sea la primera forma de la secuencia o la que, dentro de dicha secuencia, sea la que sigue a una forma voseante, presentan una distribución más afín a la distribución general de la variable tuteo voseo observada según el método de recogida de datos. En el primer contexto, hay una correspondencia total entre ambas distribuciones. En efecto, esta es de un $87 \%(\mathrm{~N}=260 / 298)$ de formas voseantes versus un $13 \%(\mathrm{~N}=38 / 298)$ de formas 
tuteantes. En el segundo contexto, hay una tendencia aún mayor al voseo, siendo la distribución de un $96 \%(\mathrm{~N}=76 / 79)$ de formas voseantes versus un $4 \%(\mathrm{~N}=3 / 79)$ de formas tuteantes. En mi opinión, esas distribuciones no son sorprendentes, sino que se inscriben en una pauta observada por Labov (1994: 550) según la cual la variación morfológica y sintáctica está grandemente influenciada por una tendencia a preservar estructuras paralelas. En el marco de este trabajo, esa tendencia resultó ser absoluta al optar los hablantes por iniciar una secuencia con dos o más formas de tratamiento para referir a la segunda persona del singular con una forma tuteante. No obstante, debido a la tendencia de los hablantes a optar por una forma voseante como primera forma de tratamiento de la secuencia, es de notar que cuento con solo nueve ocurrencias que corresponden al ordenamiento en el que la secuencia se inicia con una forma tuteante y le sigue otra forma de tratamiento. En este sentido, hace falta que estudios prospectivos con un mayor número de ocurrencias correspondientes a esa sucesión exploren la posibilidad de encontrar variación dentro de tal parámetro.

\section{OBSERVACIONES FINALES}

En este estudio, me propuse dos objetivos centrales. Primero, me planteé contrastar la frecuencia relativa con la que los hablantes del español chileno oriundos de Santiago de Chile recurren al voseo verbal en el corpus conversacional que uso en este estudio con las frecuencias relativas apuntadas en estudios que emplean corpus basados en cuestionarios de actitudes lingüísticas, interacciones conversacionales desarrolladas en medios de comunicación, notas de campo y entrevistas sociolingüísticas. Dado el carácter más natural del corpus aquí empleado y teniendo en cuenta la idea procedente de estudios previos según la cual los hablantes de la modalidad chilena de español atribuyen al voseo verbal un prestigio encubierto, el supuesto de base con el que trabajé fue que encontraría una frecuencia relativa de uso de este rasgo mayor que las frecuencias observadas en otros estudios que se basan en corpus de distinta naturaleza.

Segundo, me propuse identificar las posibles variables independientes que condicionan la alternancia tuteo $\sim$ voseo en contexto conversacional teniendo presente tanto parámetros sociales como lingüísticos y prestando especial atención a los factores que inciden significativamente en el uso del voseo verbal. A partir de estudios previos, trabajé con el supuesto de base de que el uso de formas verbales voseantes presentaría correlaciones importantes 
con las variables género, edad y especificidad del interlocutor, reforzando la idea según la cual los hablantes del español chileno asignan un prestigio encubierto al voseo verbal.

Los resultados observados permiten afirmar que ambas hipótesis son aceptables. Asimismo, permiten concluir que, si bien en el contexto más natural de la conversación los hablantes de la modalidad chilena de español oriundos de Santiago recurren al voseo en la gran mayoría de los casos en los que refieren a la segunda persona del singular, los factores sociales y lingüísticos que llegan a restringir su uso de manera estadísticamente significativa ponen en evidencia su naturaleza estigmatizada.

\section{REFERENCIAS BIBLIOGRÁFICAS}

Bates, Douglas et al. 2015. Fitting linear mixed-effects models using lme4. Journal of Statistical Software 67(1): 1-48.

Bayley, Robert. 2013. Variationist Sociolinguistics. En Robert Bayley, Richard Cameron y Ceil Lucas (eds.). The Oxford Handbook of Sociolinguistics, pp. 11-30. New York: Oxford University Press.

Bello, AndRÉs. 1884 [1834]. Advertencias sobre el uso de la lengua castellana dirigidas a los padres de familia, profesores de colegios y maestros de escuelas. En Andrés Bello (ed.). Obras Completas, pp. 468-486. Santiago de Chile: Imprenta de Pedro G. Ramírez.

Bertolotti, Virginia. 2015. A mí de vos no me trata ni usted ni nadie. Sistemas e historia de las formas de tratamiento en la lengua española en América. México, D.F. y Montevideo: Universidad Nacional Autónoma de México y Universidad de la República.

Bishop, Kelley y Jim Michnowicz. 2010. Forms of address in Chilean Spanish. Hispania 93(3): 413-429.

Carvalho, Ana Maria. 2010. ¿Eres de la frontera o sos de la capital? Variation and alternation of second-person verbal forms in Uruguayan border Spanish. Southwest Journal of Linguistics 29(1): 1-23.

Coulmas, Florian. 2005. Sociolinguistics: The Study of Speakers' Choices. Cambridge: Cambridge University Press.

DíAz-CAMpos, Manuel. 2014. Introducción a la sociolingüistica hispánica. Malden y Oxford: Wiley-Blackwell.

ECKert, Penelope. 1997. Age as a sociolinguistic variable. En Florian Coulmas (ed.). The Handbook of Sociolinguistics, pp. 151-167. Oxford: Blackwell.

FERnÁNDEZ Mallat, Víctor. 2011. El 'voseo mixto verbal' de hablantes chilenos en Montreal: Estudio de caso en un contexto de contacto dialectal. Boletín de Filología de la Universidad de Chile 46(2): 35-58.

HeLINCKS, KRIS. 2012. La variación social y estilística del voseo chileno en diferentes géneros televisivos. Revista Internacional de Lingüistica Iberoamericana 19(1): 185-211.

2015. Negotiation of terms of address in a Chilean television talk show. Bulletin of Hispanic Studies 92(7): 731-752.

Holmes, Janet. 1992. An Introduction to Sociolinguistics. London y New York: Longman. 
Kluge, Bettina. 2005. Las fórmulas de tratamiento en un corpus chileno. En Volker Noll, Klaus Zimmermann e Ingrid Neuman-Holzschuh (eds.). El español en América. Aspectos teóricos, particularidades, contactos, pp. 169-188. Frankfurt am Main y Madrid: Vervuert e Iberoamericana.

2010. El uso de las formas de tratamiento en las estrategias de generalización. En Martin Hummel, Bettina Kluge y María Eugenia Vázquez Laslop (eds.). Formas y fórmulas de tratamiento en el mundo hispánico, pp. 1107-1136. México, D.F. y Graz: El Colegio de México y Karl-Franzens-Universität Graz.

Labov, William. 1966. The Social Stratification of English in New York City. Washington, DC: Center for Applied Linguistics.

1972. Sociolinguistic Patterns. Philadelphia: University of Philadelphia Press. 1994. Principles of Linguistic Change. Volume 1: Internal Factors. Oxford: Wiley-Blackwell.

2001. Principles of Linguistic Change. Volume 2: Social Factors. Oxford: WileyBlackwell.

Morales, Félix. 1972-1973. El voseo en Chile. Boletín de Filología 23-24: 261-273.

Milroy, Lesley y Matthew Gordon. 2003. Sociolinguistics: Method and Interpretation. Malden et al.: Blackwell.

Newall, Gregory. 2007. The loss of the voseo in Chilean Spanish: Evidence in literature. University of Pennsylvania Working Papers in Linguistics 13(2): 165-178.

PINHEIRO, JosÉ et al. 2017. nlme: linear and nonlinear mixed effects models, R package version 3.1-131 [en línea]. Disponible en https://CRAN.R-project.org/package=nlme [Consulta 09/06/2017].

R CORE TEAm. 2017. R: A language and environment for statistical computing, R Foundation for Statistical Computing, Vienna, Austria [en línea]. Disponible en http://www.R-project. org/ [Consulta 09/06/2017].

Rivadeneira Valenzuela, Marcela. 2016. Sociolinguistic variation and change in Chilean voseo. En María Irene Moyna y Susana Rivera-Mills (eds.). Forms of Address in the Spanish of the Americas, pp. 87-117. Amsterdam y Philadelphia: John Benjamins.

Rivadeneira, Marcela J. y Esteve B. Clua. 2011. El voseo chileno: Una visión desde el análisis de la variación dialectal y funcional en medios de comunicación. Hispania 94(4): 680-703.

Rona, José Pedro. 1967. Geografía y morfología del voseo. Tesis de doctorado, Pontifícia Universidade Católica do Rio Grande do Sul.

Silva-Corvalán, Carmen. 1984. The social profile of a syntactic-semantic variable: Three verb forms in Old Castile. Hispania 67(4): 594-601.

Stevenson, Jeffrey Lee. 2007. The Sociolinguistic Variables of Chilean Voseo. Tesis de doctorado, University of Washington.

Sweeney, Patricia. 2005. El voseo en Chile: Factores histórico-morfológicos que explican su aparición y mantenimiento. Tesis de doctorado, Albany State University.

Tagliamonte, Sali A. 2012. Variationist Sociolinguistics: Change, Observation, Interpretation. Malden y Oxford: Wiley-Blackwell.

Torrejón, Alfredo. 1986. Acerca del voseo culto de Chile. Hispania 69(3): 677-683.

1989. Andrés Bello, Domingo Faustino Sarmiento y el castellano culto de Chile. Thesaurus 44(3): 534-558.

2010a. El voseo en Chile: una aproximación diacrónica. En Martin Hummel, Bettina Kluge y María Eugenia Vázquez Laslop (eds.). Formas y fórmulas de tratamiento en el mundo hispánico, pp. 413-427. México, D.F. y Graz: El Colegio de México y KarlFranzens-Universität Graz.

2010b. Nuevas observaciones sobre el voseo en el español de Chile. En Martin Hummel, Bettina Kluge y María Eugenia Vázquez Laslop (eds.). Formas y fórmulas de 
tratamiento en el mundo hispánico, pp. 755-769. México, D.F. y Graz: El Colegio de México y Karl-Franzens-Universität Graz.

TRudgill, Peter. 1972. Sex, covert prestige and linguistic change in the urban British English of Norwich. Language in Society 1(2): 175-195.

Wolfram, Walt, Kirk Hazen y Natalie Schilling-Estes. 1999. Dialect Change and Maintenance in Outer Banks English, Publication of the American Dialect Society 81. Tuscaloosa: University of Alabama Press. 